\title{
EL TSUNAMI FINANCIERO DE ESTADOS UNIDOS YSU IMPACTO SOBRE LA EMIGRACIÓN MEXICANA
}

\section{RODOLFO GARCÍA ZAMORA*}

1. DE LA CRISIS FINANCIERA NORTEAMERICANA A LA CRISIS FINANCIERA MUNDIAL

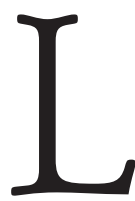
a semana del 15 de septiembre de 2008 la industria financiera global sufrió un infarto. Murió el 18 de septiembre, pero fue resucitada por el secretario del Tesoro Paulson con un choque eléctrico de alto voltaje que en última instancia representó 700 mil millones de dólares de rescate gubernamental. El origen de esta crisis se remonta a los años sesenta. Hasta esa década, la ortodoxia económica de capitalismo del siglo XIX dirigió la política monetaria y fiscal. Esa ortodoxia exigía presupuestos gubernamentales equilibrados, moneda sana respaldada por oro y un comercio internacional equilibrado. El abandono de los esos tres principios durante las cuatro décadas pasadas es una de las causas directas de la crisis del capitalismo que hoy nos amenaza.

El abandono del último principio, comercio equilibrado, se dio de manera natural a partir del rechazo de los dos primeros. El excesivo gasto gubernamental sobrestimuló la economía estadounidense y fomentó el exceso de importaciones. Y, libre del patrón oro, el país pudo financiar colosales déficits comerciales con dólares de papel y bonos del Tesoro denominados en dólares. En el 2006, el déficit de cuenta corriente estadounidense se disparó a casi 800 mil millones de dólares anuales, alrededor de 2 mdd por minuto. Ese déficit y el crédito que lo financió, desestabilizaron la economía global al crear desequilibrios insostenibles que ahora se revierten. ${ }^{1}$

Una vez abandonados los principios fundamentales del capitalismo, cualquier locura financiera concebible se convirtió en una oportunidad lucrativa para Wall Street. Como el dólar dejó de estar respaldado por el oro, la Reserva Federal perdió el control sobre la generación del crédito. La división entre dinero y crédito se hizo borrosa, y luego desapareció por completo. Los pasivos de Fannie Mae y Freddie Mac, se incrementaron a 5 billones de dólares y, a medida que su deuda se expandía, adquirieron o garantizaron más de la mitad de las hipotecas

\footnotetext{
* Doctorado en Estudios del Desarrollo, UAz. www.estudiosdeldesarrollo.net

1 «Orígenes de la crisis». Economist Intelligense Unit. La Jornada, 2 de diciembre 2008.
} 
nacionales, y elevaron el precio de los inmuebles en el proceso.

Los banqueros fraccionaron, reempaquetaron y revendieron una enorme gama de instrumentos de deuda (ahora conocidos como deuda tóxica) y pagaron a las agencias calificadoras para obtener calificaciones AAA. Se crearon y proliferaron instrumentos crediticios derivados que, según el recuento más reciente, sobrepasan 600 billones de dólares en valor teórico, el equivalente a casi 100 mil dólares por persona en el planeta.

Durante los 20 años pasados Alan Greenspan supervisó la explosión de esa deuda; su presidencia, posiblemente la peor en la historia de la Reserva Federal, mantuvo bajas las tasas de interés, desalentó la regulación, pero animó la innovación del sector financiero. Y celebró la rápida expansión de los instrumentos derivados como un medio de mejorar la estabilidad del sector mediante la distribución de riesgos hacia quienes eran más capaces de enfrentarlos. En el nivel macro, su estilo podría ser caracterizado como la administración de burbujas económicas que fueron populares durante su largo encargo. Para la posteridad, Greenspan será el actor principal en la creación de esta crisis. Muy a menudo, las entidades reguladoras facilitaron los excesos. La industria financiera se desreguló, la ley Glass-Stegall se derogó y se forjaron enormes fortunas. Los mercados de derivados se propalaron ante la falta de supervisión regulatoria; los agentes hipotecarios generaron miles de millones de dólares en hipotecas subprime, prácticamente sin ninguna vigilancia y los bancos burlaron los requerimientos de suficiencia de capital al ocultar sus activos. ${ }^{2}$

Para José A. Estévez ${ }^{3}$ en su artículo «La autorregulación en los mercados financieros» las causas de la actual crisis de los mercados financieros son varias y complejas. Entre ellas se cuentan, en primer lugar, la desregulación que ha permitido crear nuevos activos financieros, como los bonos estructurados respaldados por hipotecas de alto riego. En segundo lugar, la liberalización de los mercados de capitales y la libre circulación de éstos. En tercer lugar, la existencia de paraísos fiscales en los que los bancos de inversión crearon entidades para emitir los bonos contaminados $y$, en cuarto lugar, la ineficacia de los mecanismos de control como los bancos centrales y, en particular las agencias de rating.

Para Estévez, en algunos aspectos, la actual crisis tiene similitudes con el clásico timo de la estampita. Es como si nos vendieran un sobre cerrado diciéndonos que contiene un fajo de 500 euros. Para garantizarlo, hay un sello de una entidad de confianza. El sobre supuestamente lleno de billetes es uno de esos productos estructurados tan complejos que quienes los adquieren no sabe muy bien qué está comprando. Las entidades que pusieron el sello diciendo que efectivamente eran

2 Idem.

${ }^{3}$ Revista Mientras Tanto, Número 62, Barcelona, octubre 2008. 
billetes fueron las agencias de rating. Y los problemas empezaron cuando algunos abrieron el sobre y se dieron cuenta de que, en realidad, contenía no sólo billetes, sino también recortes de periódicos. Todo mundo quiso deshacerse de los sobres sellados, pero no encontraron a nadie que quisiera comprarlos (al menos hasta que Bush puso sobre la mesa un cheque por valor de 700 mil millones de dólares).

El crash de 2008 aconteció en un año de tres crisis distintas, pero vinculadas. Ya existía una crisis de combustibles, que elevó los precios del crudo a casi 150 dólares por barril y una de alimentos, con motines y controles a las exportaciones, mientras los precios del trigo y del arroz se triplicaban. Luego, la explosión de la burbuja inmobiliaria y el mercado de hipotecas subprime causó una crisis financiera que comenzó con una repentina caída del valor de los activos que despertó dudas sobre la solvencia de las principales instituciones bancarias. Esto provocó a su vez una crisis de liquidez, ya que el préstamo interbancario casi desapareció, lo que condujo a una restricción crediticia a medida que los temerosos bancos reducían préstamos acreditados tradicionalmente confiables de los sectores de servicios y manufacturas.

La bancarrota de Lehman Brothers, en septiembre, desencadenó el pánico. La disminución del precio de los activos y la reducción del consumo convirtió la desaceleración en una recesión que golpeó a las economías industrializadas del G-7 y luego se expandió. A principios de octubre, el mundo se encaminaba a una catastrófica quiebra del sistema bancario. El gobierno inglés tomó medidas decisivas al anunciar que respaldaría a sus bancos con crédito ilimitado y se preparó para nacionalizarlos. Los gobiernos de Estados Unidos y Alemania otorgaron respaldos similares, apoyados por los ministros de hacienda del G-7 en la reunión que se llevó a cabo en Washington del 11 al 12 de octubre. El mundo ha sorteado la amenaza inminente de una catástrofe. Un cataclismo bancario global es ahora poco probable, aunque estuvo a punto de suceder. ${ }^{4}$

Los impactos de la crisis financiera mundial de septiembre-octubre tienen implicaciones de mayor calado. En efecto, el consenso es absoluto: la peor crisis financiera global desde la gran depresión obliga a repensar la estructura de los mercados financieros internacionales y a una mayor cooperación de los principales organismos reguladores, expresaron los directivos de las más importantes instituciones financieras en el Foro Mundial del Conocimiento en Seúl. ${ }^{5}$ En el mismo sentido, los líderes de 43 países europeos y asiáticos abogaron en Pekín por una reforma completa del sistema financiero global, y llamaron al Fondo Monetario Internacional a ayudar a los países más afectados por la crisis. ${ }^{6}$

\footnotetext{
${ }^{4}$ «La vida después del crash». Economist Intelligense Unit. La Jornada, 16 de diciembre 2008.

5 «Repensar los mercados globales». Economist Intelligense Unit, 9 de diciembre 2008.

6 South China Morning Post, 25 de octubre 2008.
} 
Los impactos recesivos a nivel internacional por la crisis financiera eran ya evidentes en la segunda semana de noviembre de 2008, como lo prueban las declaraciones de la Organización para la Cooperación y el Desarrollo Económico (OCDE) en Paris el 13 de noviembre, cuando anuncia que las economías desarrolladas están en recesión y en riesgo de contraerse también el año próximo. ${ }^{7}$ Las

declaraciones anteriores son ratificadas por el Fondo Monetario Internacional en Francfort el 10 de diciembre, cuando uno de sus altos funcionarios, John Lipsky, anuncia que la crisis financiera se está convirtiendo en una verdadera depresión, azotando de igual forma tanto a los países desarrollados como a las economías emergentes. "Los riesgos de una deflación están comenzando a convertirse en una preocupación en Japón, Estados Unidos y las brechas en la producción se han ampliado». Las economías desarrolladas necesitan prepararse para una importante recesión, alertó.

Las recesiones en varias economías avanzadas podrían durar más tiempo que en situaciones anteriores, posiblemente justificando una mayor inversión pública en proyectos que típicamente tienen una duración más larga pero acarrean beneficios sustanciales a largo plazo. ${ }^{8}$

A nivel de los impactos de la crisis financiera mundial sobre el empleo, la
OCDE, a través de su secretario general, José Ángel Gurría, expresa que la crisis económica mundial dejará sin trabajo a 20 millones de personas, lo que equivaldrá a más del 10\% global en $2010 .{ }^{9}$

Sobre los impactos globales de la crisis financiera mundial sobresale, en diciembre, la colaboración de León Bendesky, «El gran vuelco», ${ }^{10}$ donde destaca cómo la crisis financiera desatada en Estados Unidos ha desquiciado los mercados de dinero y capitales, la producción, el empleo y el consumo. Además, ha provocado una enorme expansión de la deuda del gobierno, así como formas de intervención en las empresas privadas sin parangón. Para él, el sistema financiero tal como existía hace apenas nueve meses, es hoy irreconocible en cuanto a su estructura institucional; los instrumentos convencionales del crédito han desaparecido y las corrientes de los préstamos de crédito han dejado de operar para fines prácticos. Todo esto ocurre a pesar de las enormes inyecciones de dinero por parte el Tesoro y de la Reserva Federal.

Bendesky indica que la política monetaria ha llegado a su límite una vez que las tasas de interés de los títulos de deuda de corto plazo del gobierno tienen tasa cero. Los ahorradores se refugiaron por ahora en ese tipo de deuda, aún con rendimientos reales (luego de la inflación) negativos; lo hacen por la

7 La Jornada, 14 de noviembre 2008.

8 La Jornada, 11 de diciembre 2008.

9 La Jornada, 16 de diciembre 2008.

${ }^{10}$ La Jornada, 15 de diciembre 2008

136 PRIMER SEMESTRE 2009 MIGRACIÓN Y DESARROLLO 
garantía del gobierno federal. Por otro lado, resalta que no se ha superado todavía el riesgo de una posible deflación, es decir, la caída de los precios que agravará aún más la situación recesiva. La corrección de los precios de los bienes raíces no se ha dado y las presiones hacia la contracción del producto persisten. La muy frágil condición de las tres grandes empresas automotrices agrava las cosas y su efecto se extiende por una larga cadena de actividades subsidiarias dentro y fuera de Estados Unidos.

A nivel de propuestas, enfatiza cómo ahora se pone cada vez más atención en las políticas de estímulo fiscal sobre la demanda agregada mediante el gasto público en una serie de rubros, especialmente la infraestructura física y energética del país como lo plantea Obama. Sin embargo, tales políticas son problemáticas y muy debatidas. No hay una clara teoría económica que ampare este tipo de intervención en cuanto a su efectividad para salir de modo rápido de la depresión económica en curso. Se cita mucho a Keynes y las políticas del Nuevo Trato de Roosevelt, pero la situación de los años de 1930 era distinta en muchos sentidos. Uno es la extensión y profundidad de las relaciones económicas globales; otro es que finalmente la crisis de aquella época se superó sólo luego de que la economía de guerra se transformó en civil hasta 1945.

Ahora que se recurre al gasto público como el antídoto infalible ante la recesión, antes condenado durante 30 años, señala, que es ciertamente, un estímulo necesario; es más, casi único en las condiciones actuales, pero no tiene un efecto automático en el funcionamiento y la corrección de los mercados. La asignación de ese gasto y la manera en que se transmite en los diversos canales de la economía ocurre de manera compleja y con diversos obstáculos y cuellos de botella. La efectividad de este tipo de gasto no está asegurada.

Respecto al impacto de la expansión fiscal, Bendesky señala que no se puede prever con certeza cuál será su resultado sobre el nivel de la actividad económica, ni cuánto tiempo tarde en cumplir el objetivo que se le asigna. A eso hay que sumarle la fuerte ampliación de la deuda pública en que se ha incurrido, así como la depreciación de los activos de las familias, en especial en sus viviendas y su alto nivel de endeudamiento. Las deudas habrá que pagarlas en algún momento. Una forma de reducir la carga de la deuda es la inflación, pero en el marco actual eso no es posible. Este es uno de los dilemas actuales del entorno político, de las políticas públicas y de los difíciles acuerdos que tienen que establecerse so pena de provocar rupturas graves en un entorno social por demás debilitado.

El autor antes referido indica que el vuelco provocado por esta crisis ha puesto en evidencia las concepciones teóricas e ideológicas, así como las prácticas de la gestión estatal que prevalecieron durante tres décadas. Esas concepciones se derrumban. Es 
una interesante paradoja que esto le haya sucedido a una administración como la de Bush con su explícita y provocadora defensa y promoción de un liberalismo a ultranza. Este periodo ha estado lleno de fraudes financieros como Enron, Halliburton y el más reciente de Madoff por $54 \mathrm{mil}$ millones de dólares al final del 2008. La realidad muestra que ya no puede sostenerse más la capacidad intrínseca de ajuste de los mercados, pero, tampoco puede sostenerse el automatismo de la intervención estatal. Esta situación no es, sin embargo, un callejón sin salida. Pero su superación no va a encontrarse en los cubículos de los profesores ni en los corredores del poder político esclerotizado.

El restablecimiento de un acuerdo social funcional será imprescindible. Para que esto ocurra deberá haber condiciones materiales mínimas y liderazgos efectivos que canalicen la presión social. Las resistencias de todas las partes involucradas van a ser muy grandes en una estructura de poder tan estrechamente vinculado. Se abre un periodo de contradicciones con fuertes fricciones que no se resolverán en el ámbito de los intereses nacionales definidos estrechamente. ${ }^{11}$

\section{LOS IMPACTOS ECONÓMICOS SOBRE MÉXICO}

Al inicio de la crisis financiera en Estados Unidos, las autoridades mexicanas de la Secretaría de Hacienda y Presidencia de la República subestimaron los impactos que ella podría generar en la economía nacional por estar «blindada» contra impactos negativos y que en todo caso podrían ser impactos leves equivalentes a un «resfriado» o un «catarrito». Sin embargo, la fuerza de los hechos obligó a tales autoridades a reconocer la vulnerabilidad económica y del país y el 8 de octubre de 2008 el presidente Calderón da a conocer el Programa para Impulsar el Crecimiento y el Empleo, orientado a «mitigar el impacto negativo en la economía mexicana de la turbulencia financiera internacional». El plan establece la ampliación del gasto público, particularmente en materia de infraestructura; la modificación de las reglas en el ejercicio del presupuesto para agilizar la inversión oficial; la construcción de una nueva refinería, financiada con 12 mil millones de pesos acumulados del Fondo de Estabilización de Petróleos Mexicanos; un «programa extraordinario» de apoyo a las pequeñas y medianas empresas, y las medidas para desregular y desgravar importaciones para «hacer más competitivo al aparato productivo nacional».

Este Programa constituye un indicio de entendimiento, por parte de la actual administración, de la gravedad de la problemática que enfrenta el país; una variación, por pequeña que sea, con respecto al férreo empecinamiento neoliberal de los últimos años. Sin embargo,

11 Idem. 
El Programa es tardío e insuficiente: las medidas ahora propuestas, más otras de mayor calado, habrían tenido que aplicarse por lo menos desde la pasada sucesión presidencial. El tiempo perdido implica que la crisis mundial encuentra una economía mexicana débil, dependiente, estancada y distorsionada por decisiones políticas equívocas o, simplemente, por falta de decisiones. Este estímulo coyuntural a los motores internos de la economía no remedia los efectos de la falta de incentivos como uno de los más graves vicios crónicos del ciclo de gobiernos neoliberales. ${ }^{12}$

Los impactos de la crisis financiera en México también son reconocidos por Guillermo Ortiz, titular del Banco de México, en Washington, el 12 de octubre cuando expresa que la crisis financiera internacional ya le ha hecho «bastante daño a la economía mexicana». «La devaluación del peso brincó por donde menos esperábamos, estamos en un tsunami financiero que afecta a toda la gente». ${ }^{13}$ La misma Secretaría de Hacienda reconoce a finales de septiembre que los impactos recesivos de la crisis financiera a nivel de empleo provocarán que sólo sean generados en el país de 350 a 400 mil nuevos empleos en el 2008. ${ }^{14}$

Para León Bendesky, ${ }^{15}$ una vez que se reconocen los impactos negativos de la crisis financiera internacional sobre México, hoy resulta imprescindible empezar a replantearse el modo como funciona la economía mexicana. Lo peor que puede pasar es que tras el largo periodo de dificultades que está por delante se piense que todo puede seguir como era antes. Esto tiene que ver con la estructura del sistema financiero, con las relaciones comerciales, con la concentración del mercado, de la riqueza y del ingreso, con las pautas de la desigualdad prevalecientes. Este es el momento de establecer medidas de protección ante la crisis y, al mismo tiempo, plantearse una estrategia de transformación que se asocie con las inversiones productivas, con la gestión fiscal, con la dinámica demográfica y del empleo, con la definición de los patrones de productividad y de la competitividad internacional y con el desarrollo regional. México es un país que se ha rezagado sensiblemente en su situación interna y en su posición externa. Para superar ese atraso tiene que sacudirse y superarse el pasmo del gobierno, la desidia de los organismos empresariales y la restringida participación de las organizaciones sociales.

Los impactos de la crisis financiera internacional rebasan el ámbito financiero y el mismo Banco Mundial alerta que la recesión económica mundial afectará a los grupos más pobres en México, donde hay riesgos de desempleo adicional. ${ }^{16}$ Información que es corroborada por Julio Boltvinik

12 La Jornada, 10 de octubre 2008.

${ }^{13}$ La Jornada, 13 de octubre 2008.

${ }^{14}$ La Jornada, 27 de octubre 2008.

${ }^{15}$ La Jornada, 20 de octubre 2008.

${ }^{16}$ El Universal, 23 de octubre 2008. 
cuando informa que la actual crisis financiera y económica global podría empujar a 10 millones de mexicanos más a la pobreza durante al año entrante. Para él, la cifra se suma a los 6.5 millones de pobres que han generado las alzas en los precios de alimentos desde el 2006. En consecuencia, para el 2009 aproximadamente 64.5 millones de mexicanos formarían parte de lo que la Secretaría de Desarrollo Social (Sedesol) define como pobreza de patrimonio, es decir, la población que no tiene cubiertas «aquellas necesidades que permiten al ser humano vivir de manera digna». La experiencia de México muestra cómo en las crisis de 1994-1995 y en la de 1982-1984, la pobreza se incrementó entre 10 y 12 puntos porcentuales. ${ }^{17}$

En el mismo sentido, el Consejo Nacional de Evaluación de las Políticas Sociales informa a los senadores que la pobreza crecerá este año y el próximo, debido al incremento en los precios de los alimentos y por la crisis financiera mundial. Señala, además, que la desigualdad sigue siendo muy alta "y no ha mejorado sustancialmente en 14 años, periodo en el que sólo se redujo en dos puntos la concentración del ingreso, entre $10 \%$ de la población más rica del país, quienes pasaron de retener $41.6 \%$ de la riqueza nacional a $39.3 \%$ entre 1992 y 2006». ${ }^{18}$

La situación anterior contrasta con el informe del Banco de México de inicios de diciembre respecto a cómo los empresarios mexicanos, al amparo de la estabilidad cambiaria, sacaron del país poco más de 19 mil millones de dólares en nueve meses para depositarlos en cuentas bancarias o adquirir empresas en el extranjero. ${ }^{19}$ Esta actuación empresarial de sacar su dinero del país es corroborada por el informe de Banco Bilbao Vizcaya Argentaria Bancomer respecto a cómo los inversionistas externos sacaron del país 22 mil 190 millones de dólares, monto adicional a los 18 mil 277 millones de dólares que usó el Banco de México para hacer frente a las presiones devaluatorias. ${ }^{20}$

Para inicios del mes de diciembre existe consenso en que el comportamiento de la economía nacional en el 2009 estará por debajo de las expectativas hechas meses atrás. En efecto, el optimismo que había se acabó. El próximo año se vislumbra como uno de los peores en la última década, con un estancamiento en el crecimiento del Producto Interno Bruto (PIB), magra creación de empleos, una inflación no vista desde diciembre del 2001 y un desplome en el índice de confianza.

Por primera vez durante 2008, en la encuesta del Banco de México los analistas económicos del sector privado prevén que en el primer trimestre de 2009 el PIB decrecerá $0.1 \%$, y que en todo ese año sólo crecerá $0.38 \%$, rango nunca antes

17 Idem.

${ }^{18}$ La Jornada, 5 de diciembre 2008.

${ }^{19}$ La Jornada, 3 de diciembre 2008.

${ }^{20}$ La Jornada, 18 de diciembre 2008.

140 PRIMER SEMESTRE 2009 MIGRACIÓN Y DESARROLLO 
visto en la historia reciente. Lo menos que había crecido la economía nacional en un año fue $0.8 \%$, en 2002 . También anticipan que al cierre de 2009 la inflación será de 4.4\%, cifra idéntica a la de diciembre de 2001, por lo que el banco central no podrá alcanzar su meta antes de los próximos siete años. La perspectiva del Banco de México, de converger hacia su meta del 3.0\% hacia el cierre de 2010, parece diluirse en el tiempo, toda vez que entre 2009 y 2012, los analistas estiman una inflación promedio anual de $3.97 \%$ y de 2013 a 2016, el promedio es de $3.49 \%$. Mientras tanto, el número de empleos formales que se crearían en 2009 sería de 161 mil, apenas 16.1\% del que exige la población, y ligeramente por arriba de los 122 mil 852 empleos creados entre octubre de 2007 y $2008 .{ }^{21}$

Según Alejandro Nadal, ${ }^{22}$ la crisis ha generado una doble discusión en México. La primera es sobre los efectos del colapso económicofinanciero internacional. La segunda parte del debate se concentra en la forma de enfrentar la crisis. El gobierno sostiene que ya ha presentado un plan de acción. Los críticos piensan que está sumergido en la negación y pasividad. Quizá es bueno situar esta discusión en una perspectiva más profunda.

La crisis financiera y económica global no será como las recesiones que han marcado a jalones la economía de Estados Unidos en los últimos 30 años. Durará unos 20 meses, quizá más. Estados Unidos hasta podría sumergirse en un proceso más largo de estancamiento.

¿Por qué va a durar tanto la recesión? Porque ahora se presentan varios círculos viciosos entrelazados y se necesita más tiempo para que la política económica pueda romperlos. Por otra parte, la crisis se extendió geográficamente y eso hace más difícil la recuperación sin una coordinación internacional.

Desde esa perspectiva, ¿̇Cómo se presentan las cosas para la economía mexicana? Para Nadal, la primera consecuencia es que habrá un menor crecimiento. La meta corregida por el gobierno para la expansión del PIB 2009 es de $2.5 \%$. Hoy las estimaciones más frecuentes están más cerca de los números negativos, entre cero y $-0.5 \%$. Es probable que el PIB se contraiga $-1.5 \%$

Los efectos sobre el empleo serán devastadores. La tasa mensual de desempleo abierto para el mes de noviembre (4.47\% de la PEA) es el nivel más alto en tasas mensuales desde enero de 1997, lo cual nos regresa a la cúspide de la crisis de 1994-1995. Para una economía que hoy cuenta con 12 millones de personas ocupadas en el sector informal (datos del INEGI) la pérdida de empleos formales es muy mala noticia. El salario real seguirá su caída secular, lo cual no augura nada bueno para México. El desempleo agravará la cartera vencida y la posición morosa de muchos deudores

${ }^{21}$ El Financiero, 2 de diciembre 2008.

22 La Jornada, 24 de diciembre, 2008. 
explotados por una banca ciega y voraz.

Mucho se habla de las operaciones de cobertura realizadas por la Secretaría de Hacienda contra el riesgo de reducciones en el precio del petróleo. Buena medida, pero no frenará la caída en los ingresos tributarios debido a la contracción económica. Por eso no hay que descartar un episodio más de los temidos ajustes fiscales en 2009.

En el sector externo, para el autor, las cosas no pintan bien por la reducción de exportaciones y de remesas. EL sector maquilador sufrirá en sus tres componentes medulares (autopartes, electrónica y textil/confección). Si el desequilibrio externo no crece más, es porque la economía mexicana estará sumergida en la recesión.

Los indicadores anteriores revelan con claridad que la economía necesita tratamiento urgentemente. El gobierno anunció en octubre un plan anticrisis que es una broma de mal gusto. No dice nada sobre política monetaria, y el «estímulo» fiscal es un simple arreglo de partidas por la redefinición del régimen de inversiones de Pemex.

Es cierto, el gobierno carece de un plan para enfrentar la crisis $y$, en cambio, continúa adoptando medidas que profundizarán y alargarán los efectos nocivos del colapso económico. Ni se ha abaratado el crédito, ni se han reducido los impuestos al consumo, ni se ha generado un programa fiscal que pudiera generar un impulso masivo al empleo.

Ante lo anterior. Surgen dos preguntas. Primero. ìSe puede tener un plan anticrisis con este modelo? La respuesta es negativa. El modelo económico aplicado aquí es, básicamente, el mismo que provocó la crisis de 1995 y sólo funciona para beneficiar al capital financiero. Por eso el Banco de México se niega a reducir la tasa de interés. Poco le importa sacrificar empresas y empleo. Su prioridad es la estabilidad cambiante y una rentabilidad "competitiva» para el capital financiero. Bajo las reglas de este modelo neoliberal, no se puede tener una política monetaria anticíclica. La economía real puede irse a freir espárragos.

Lo anterior nos lleva a la segunda pregunta. ¿̇Para qué se quiere un paquete de estímulos frente a la crisis? ¿Para regresar al lugar donde estábamos? Si esa es la respuesta, estamos fritos. La economía mexicana tiene 25 años de permanecer en un estado de semiestancamiento. Entre 1983 y 2008, en promedio el PIB ha crecido sólo $2.4 \%$ cada año. En ese lapso, México siguió sufriendo crisis por desequilibrios en las cuentas externas y un estado lamentable de las finanzas públicas. El rezago en educación, salud, vivienda, ciencia y tecnología, y medio ambiente es colosal. Se sacrificó una generación debido a un modelo económico que no funciona. Hasta la viabilidad de este país está en entredicho.

Para Alejandro Nadal, lo que se necesita no es un simple "paquete 
anticrisis». Lo que urge es una transformación profunda de estrategia y una redefinición acorde de los instrumentos de política económica, a nivel macro y sectorial. Así como están las cosas, el país no va a ninguna parte. $\bigcirc$ bueno, quizás sí, al abismo.

El 7 de enero el presidente Felipe Calderón presentó el Acuerdo Nacional a Favor de la Economía Familiar y el Empleo que, a su decir, está orientado a «superar con mayor rapidez los efectos de la situación económica adversa». El programa gira en torno de cinco ejes principales -apoyo al empleo, la economía familiar y las pequeñas y medianas empresas; inversión en infraestructura y eficiencia del gasto público- y consiste en 25 acciones, entre las que destacan la congelación de precios de las gasolinas durante todo el año; reducción del 10\% del gas LP, baja en las cuotas de las tarifas eléctricas, la asignación de recursos adicionales a Petróleos Mexicanos (Pemex) y a las entidades federativas -17 mil y $14 \mathrm{mil}$ millones de pesos, respectivamentepara inversión y desarrollo de infraestructura, así como acciones para que el gasto público se ejerza de forma «más transparente, más eficiente y con mayor oportunidad».

El plan anticrisis constituye un indicio de mínima comprensión por el actual gobierno de la gravedad de la problemática que enfrenta el país, y en este aspecto constituye un cambio en relación con la indolencia y la cerrazón exhibida antes sobre la profundidad de los efectos de la coyuntura mundial en la economía mexicana: todavía el pasado 6 de enero, citando datos de la firma de inversión Merrill Lynch, Calderón señaló que México tiene menor riesgo financiero que países como Japón, Reino Unido y Estados Unidos, en un reiterado intento de minimizar, de cara a la opinión pública, los efectos de la crisis económica internacional.

Adicionalmente, en el referido plan, se prevén medidas como el fomento al empleo mediante inversión en infraestructura, que en alguna medida rompen con el férreo neoliberalismo característico de las tres administraciones anteriores y la actual, y reconocen la importancia de la intervención del sector público en la economía.

Sin embargo, el programa anticrisis calderonista resulta tardío, pues algunas de las medidas propuestas, y otras que no se consideran, tendrían que haberse puesto en marcha desde hace tiempo. Es el caso del anuncio de congelar los precios de las gasolinas, que habría tenido mayor sentido si se hubiera aplicado, por lo menos, a principios del año pasado, antes de los 33 aumentos decretados durante los últimos doce meses. En la circunstancia actual, en cambio, el grupo gobernante ha fijado para el resto del año un costo elevado y ofensivo para la economía popular, que no tiene correlación con los pecios internacionales del petróleo -al día de hoy, el precio de este combustible es mayor en el país que hace seis meses, cuando el crudo se cotizaba en un nivel tres veces superior al actual-y 
que prácticamente ha significado un nuevo impuesto para los consumidores, fijado a discreción por la Secretaría de Hacienda. Se necesita reducir el precio de las gasolinas junto con el de otros combustibles, como el diesel-cuyos constantes incrementos han detonado manifestaciones de descontento como el paro de pescadores-, que no se encuentran en el acuerdo anunciado.

Otro aspecto cuestionable del programa se refiere a los descuentos en las tarifas del servicio eléctrico, diseñados con la clara intención de favorecer más a los grandes consumidores industriales que a los pequeños y medianos usuarios, que son quienes resienten más los efectos de las crisis económicas y quienes, por ende, debieran recibir mayores estímulos. Por lo demás, el plan contiene acciones, como el buen ejercicio del gasto público, cuya observancia debiera ser la regla en cualquier situación, no sólo en época de crisis. No se mencionan, en cambio, medidas orientadas a disminuir el gasto corriente, los salarios onerosos y las prebendas con que se regalan los altos funcionarios de la administración pública, que constituyen un importante dispendio de recursos públicos. En suma, el plan calderonista se produce fuera de tiempo y es insuficiente para contrarrestar los efectos de la actual crisis económica internacional; ésta, a fin de cuentas, se encontró en México con una economía debilitada y dependiente y una sociedad hundida en profundas desigualdades y rezagos, muy anteriores a la presente coyuntura, las cuales también fueron soslayadas en el Palacio Nacional. ${ }^{23}$

Para León Bendesky, ${ }^{24}$ el plan de apoyo a la economía que presentó el gobierno el pasado 7 de enero no es en realidad un Acuerdo Nacional. Nadie más del conjunto de la sociedad estableció ningún compromiso ante la crisis. Los gobernadores de los estados mantuvieron una actitud pasiva, el sector privado sólo demandó la modificación del impuesto empresarial de tasa única, los sindicatos y otras organizaciones sociales siguen en una posición marginal.

El plan expone, igualmente, una debilidad institucional que debe corregirse rápidamente. Uno de los actores clave de la política económica, que es el Banco de México, aparece como testigo. Una cosa es que el banco central tenga un estatuto de autonomía y otra, muy diferente, que participe de manera tangencial en la concepción de los asuntos públicos y en su expresión política. Esta disfuncionalidad es insostenible, pues en medio de esta crisis la política monetaria y su relación con los asuntos fiscales están estrechamente entrelazadas.

El acuerdo, tal como está planteado, ofrece una visión muy limitada de la magnitud de la recesión que ya se ha instalado. Una acción de gobierno es imprescindible en este momento, y los instrumentos que se proponen tendrán alguna repercusión en el comportamiento de la economía

${ }^{23}$ La Jornada, 8 de enero 2009.

${ }^{24}$ La Jornada, 12 de enero 2009. 
en los próximos meses. No

será decisivo. Y lo que se alcance dependerá de la manera en que se ejecute a lo largo de los cinco ejes que lo componen. El acuerdo no funciona en un vacío político, y en la medida en que la crisis se profundice y alargue habrá una creciente restricción de los recursos disponibles y mayor presión para disponer de ellos entre los diversos grupos de la sociedad. Esto ocurrirá en el marco de una fuerte desigualdad real y, también, de la capacidad de las demandas sobre el Estado por parte de diversos grupos.

La Secretaría de Hacienda proyecta ahora una tasa de crecimiento cero para 2009. Ese será el mejor de los casos posibles, pero es improbable. La economía de Estados Unidos sigue decayendo bruscamente en su actividad productiva y la creación de empleos; el sector financiero sigue en un fuerte desorden que se manifiesta en la ausencia de crédito, la reducción de la tasa de interés y de los precios de las acciones y de una serie de activos. La estimación de crecimiento en ese país es actualmente cercana a menos $2 \%$ para 2009. El impacto aquí va a ser mucho más grande del esperado por el gobierno. El consumo interno y la inversión no van a sostener los mercados, la creación de empleos será muy escasa, si acaso se logra, y los niveles de endeudamiento de las empresas grandes y de las familias impactarán en el uso de los recursos escasos. Ante una expectativa más realista de una fuerte caída del producto, el acuerdo reciente pronto va a perder cualquier efectividad que se le haya asignado en el marco de la política pública.

Según Bendesky, el gobierno parece no estar preparado para un escenario de este tipo, y mantiene una concepción ideológica y una práctica para conducir la economía que ya no se corresponde con las nuevas circunstancias de rompimiento del modo de acumulación anterior. Por ello hay acciones contradictorias como querer apoyar la planta productiva, y al mismo tiempo seguir eliminando los aranceles a las importaciones en un momento en que ya no tiene justificación. La falta de congruencia en las definiciones de política pública es un asunto suficientemente grave en sí mismo y, sobre todo, en las circunstancias actuales. Además de que se agrava con la incompetencia de los funcionarios del gobierno en áreas clave de la gestión de la economía. El gobierno actúa como si el problema que se enfrenta dependiera sólo de los factores que se presentan actualmente, lo que se llama de coyuntura, misma que una vez transitada nos devuelva a la situación original. Eso es falso. Por otra parte, tampoco es deseable.

Para él, se ha perdido una nueva oportunidad de replantear como funciona esta economía a partir del reconocimiento de que exhibe un muy lento crecimiento de manera crónica y de largo plazo, que la productividad y la capacidad competitiva se reducen, que prevalece una gran inequidad social y que las supuestas condiciones de fortaleza fiscal y financiera son en 
realidad endebles. Se evadió, otra vez, la ocasión de iniciar un

replanteamiento del modo de crecimiento y desarrollo de la economía mexicana, y se desperdició un momento en que el Estado podía recuperar cierta fuerza política frente a otros poderes de facto en esta sociedad. Esto va a ser muy costoso para el gobierno, pero más aún en términos colectivos.

El cuestionamiento del modo en que funciona el capitalismo y su replanteamiento va a ser ineludible a escala global. Es más, el proceso ya está echado a andar en las medidas de contención de la crisis aplicada en muchos países, sobre todo en Estados Unidos. Y van a seguir al replantear las condiciones internas de regulación de los mercados, incluyendo por supuesto la cuestión laboral. La falta de visión e iniciativas que muestra el gobierno, pero también los pocos pero muy grandes empresarios privados, los partidos políticos y los sindicatos es muy expresiva, para Bendesky, de las fuertes amarras políticas e institucionales que prevalecen en el país.

Los impactos de la crisis financiera norteamericana sobre el bajo crecimiento de la economía mexicana son más notorios a partir de la segunda semana de enero del 2009. Así, los principales grupos financieros en el país comienzan a reducir sus estimaciones de crecimiento de la economía nacional para este año, al situarlas en un escenario de contracción que oscila entre 0.2 y 0.7\% del Producto Interno Bruto (PIB), después de conocer las opiniones del gobernador del Banco de México,
Guillermo Ortiz, en el sentido de que son optimistas los pronósticos de la Secretaría de Hacienda de que habría crecimiento «cero» en 2009. El secretario de Hacienda, Agustín Carsten, señaló el 8 de enero que la economía mexicana no crecerá en 2009. Así, el pronóstico central del gobierno para el crecimiento del PIB se desplomó a $0 \%$ desde su anterior estimación de 1.8. El 9 de enero, el gobernador del Banco de México, Guillermo Ortiz, hizo importantes declaraciones en su presentación en el seminario Perspectivas Económicas 2009, organizado por el Instituto Tecnológico Autónomo de México. Allí refirió que el desempeño de la actividad económica es preocupante y que el Banco de México estima el crecimiento para este año por debajo del $0 \%$.

Por su parte, el Grupo financiero Banamex, filial de Citigroup, ajustó su previsión a un decrecimiento de $0.2 \%$. «Este ajuste a la baja del pronóstico de crecimiento del PIB del gobierno era esperado, debido al deterioro en Estados Unidos y, como resultado, de las expectativas de crecimiento para México en los últimos meses». A su vez, el Grupo Financiero Santander consideró que la desaceleración de la economía mexicana como consecuencia de la evolución desfavorable de la economía estadounidense será mayor, particularmente por las expectativas negativas que se tienen para la industria automotriz. Ante esto, al igual que las autoridades, «reconocemos que los impactos externos están siendo mayores a los 
previstos». Por ello, «reducimos nuestros pronósticos para el PiB en 2009 a una contracción de $0.7 \%$, en lugar de nuestro estimado previo de un crecimiento moderado de $0.6 \% » .^{25}$

Pero, los impactos negativos sobre la economía mexicana no sólo se perciben al inicio de enero en el menor crecimiento del PIB, sino de manera particular en el desempleo. Es así como, de acuerdo con las cifras del Instituto Mexicano del Seguro Social (IMSS), entre octubre y diciembre del año pasado se perdieron alrededor de 413 mil empleos -entre permanentes y eventuales- en los entornos urbanos del país, lo que constituye la peor caída en la ocupación formal en las pasadas tres administraciones. Esta cifra apuntala la percepción generalizada de que -no obstante el desbordado optimismo de los funcionarios del gobierno federal durante el año pasado y a pesar de las negativas a reconocer la gravedad de la actual coyuntura- los efectos de la crisis originada en el sistema financiero estadounidense se han manifestado desde hace meses en la realidad nacional, y lo han hecho en una de sus formas más severas: con la pérdida de decenas de miles de empleos y consecuente zozobra e incertidumbre para otras tantas familias. Cabe mencionar, como botón de muestra de las afectaciones derivadas de los descalabros financieros planetarios, que la industria manufacturera del país -sector altamente dependiente de los ciclos económicos de la nación vecinaacusó, durante el año pasado, un desplome de $11.3 \%$ en su producción, lo que significó el recorte de $8.22 \%$ de su personal.

A juzgar por las proyecciones realizadas por las autoridades del gobierno federal-que han pronosticado, en el mejor de los casos, un crecimiento de $0 \%$ para la economía del país en 2009-, es de suponer que las consecuencias de la presente crisis económica planetaria aún no se han expresado en toda su crudeza, y que faltan por venir meses sumamente difíciles, acompañados de una disminución más severa del mercado laboral, más desempleo y mayor crecimiento del sector informal. Debe considerarse, también, el eventual retorno de connacionales que laboran en Estados Unidos, a consecuencia de la reducción en las oportunidades de empleo en aquel país y de la intensificación de las prácticas de persecución de indocumentados por las autoridades estadounidenses. Esto significa no sólo una caída significativa en las remesas en dólares -que al día de hoy constituyen la segunda fuente de ingresos nacionales-, sino también el engrosamiento de las filas de la desocupación. ${ }^{26}$

A mediados del mes de enero, León Bendesky, ${ }^{27}$ resalta cómo cada vez más se resienten los efectos de la

\footnotetext{
25 Idem.

${ }^{26} \mathrm{Idem}$.

${ }^{27}$ La Jornada, 17 de enero 2009.
} 
crisis financiera mundial en la economía mexicana. El nivel de la actividad productiva se reduce especialmente en el sector manufacturero y cae fuertemente el flujo de las exportaciones; ello impacta adversamente la creación de empleos. Se advierte un debilitamiento general de la demanda interna que refuerza las condiciones recesivas. El gobierno ha propuesto aplicar diversas medidas de índole fiscal para contener dichos efectos negativos. Este es el caso del recientemente anunciado Acuerdo Nacional a Favor de la Economía Familiar y el Empleo; que puede preverse que sea insuficiente y deba ser complementado pronto por otras formas de intervención pública.

Bendesky destaca el debilitamiento del valor del peso en los últimos meses, además del aumento en las tasas de interés y los precios. Así, se han puesto en entredicho las bases de la estabilidad financiera que había sido la situación de la que más gala se hacía en cuanto a política económica, aún y cuando ella contrastaba con la falta de un mayor crecimiento del producto. Hoy, las condiciones financieras se han deteriorado aún más por la contracción del crédito y el aumento de su costo para las empresas y consumidores. Crece la cartera vencida y es previsible que aumenten las dificultades de pago de deudores. Este año habrá una tendencia a agravar los factores negativos en el desempeño económico y la política pública deberá orientarse de manera más activa a contenerla.

Sobre el campo monetario, indica que uno de los instrumentos con los que cuenta el Banco de México para orientar la determinación de la tasa de interés, es decir, el costo del crédito en el mercado, es fijar un objetivo para las tasas interbancarias, las que funcionan justamente entre los bancos, a corto plazo (un día). Esa es una de las condiciones que prevalecen en las transacciones de dinero y sobre las cuales tiene injerencia el banco central. Dicha tasa había llegado a $8.25 \%$. Pero El 16 de enero, las autoridades monetarias la redujeron a $7.75 \%$, indicando con ello que están dispuestas a sostener una reducción del conjunto de las tasas. Esto quiere decir, bajar el costo del crédito entre bancos, y así, reducir las restricciones que existen en el mercado de préstamos. El fundamento de esta medida significa en efecto liberar recursos y expandir la cantidad de dinero para aliviar los efectos contraccionistas de una caída de lo que se denomina como demanda agregada, en especial, el gasto en inversión y en consumo. El sustento de la baja de las tasas, según el anuncio de la política monetaria del 16 de enero es, precisamente, buscar un efecto contrario al que está generando en la economía la profundización de la crisis financiera internacional. En muchos países, como ocurre en Estados Unidos, en la Unión Europea y Japón, los bancos centrales están reduciendo las tasas de interés para intentar detener la caída 
de la demanda y contener la recesión. Eso sucede en un entorno en el que están cayendo los precios (por la misma contracción del gasto) y en el que algunos analistas prevén que puede incluso llegarse a un escenario de deflación (caída de precios). En esa circunstancia la política monetaria se hace prácticamente inefectiva.

Para Bendesky, el Banco de México apoya la medida de bajar su objetivo de la tasa de interés en la expectativa de que la inflación aquí también se va a reducir en los próximos meses y por las mismas razones de la caída de la demanda. Se busca, así, mitigar la crisis y crear estímulos para la ampliación del crédito. La jugada se vincula con el tipo de cambio. En una primera instancia podría preverse que ante menores rendimientos internos, los ahorradores podrían preferir vender pesos y comprar dólares. Eso presionaría el nivel de las reservas internacionales, o bien, depreciaría al peso frente al dólar con un efecto inflacionario que contrarrestaría la rebaja de las tasas de interés. La apuesta parece ser que hoy irse a dólares representa un alto costo puesto que los rendimientos de las inversiones en esa moneda son muy bajos y en algunos casos negativos una vez descontado el aumento de los precios. Aunque persiste la demanda de dólares como refugio soportado por la garantía que todavía representa el gobierno estadounidense.

Sin embargo, para el autor antes citado, se requiere que la estructura financiera para que la medida del banco central funcione, especialmente en el segmento bancario, responda ampliando los créditos y ajustando el costo a la baja. Esto no puede ocurrir, puesto que el $80 \%$ de la cartera de créditos está concentrado en los bancos extranjeros que hoy enfrentan grandes problemas en sus países de origen y a los que supeditan sus operaciones en el mercado local. La medida del banco central está sujeta a diversos supuestos sobre el comportamiento general de la economía, las repercusiones que provoque aquí la recesión en Estados Unidos, el curso de la inflación, las decisiones de los bancos y no menos relevante, el estado de confianza de los inversionistas y consumidores. Ante una fuerte desaceleración de la actividad económica y con pérdida de empleos, el efecto de un menor costo del crédito puede ser nulo como instrumento para contener la crisis.

Por su parte, Francisco Suárez Dávila, ${ }^{28}$ plantea que la banca se ha convertido nuevamente en uno de los principales temas políticos nacionales. Errores del gobierno o de la banca podrían magnificarlos. El sistema financiero es uno de los talones de Aquiles de nuestro mediocre crecimiento. Se evidencian las limitaciones de acción para el único gran país cuya banca casi es de propiedad extranjera. Responde a los objetivos de corto plazo de sus dueños, no al desarrollo nacional. Cuando hay, además, una crisis 
financiera mundial que afecta a sus matrices, las contradicciones son mayores. Para apoyarlas, se necesita «ordeñar» más utilidades de México y presionar más nuestro sistema cambiario. Las autoridades han actuado con debilidad hacia ella y sus abusos. No se necesitan más leyes, se requiere regulación, supervisión, políticas y visión de Estado.

1. La crisis expone los errores de nuestro "nuevo modelo bancario», copia de lo extranjero. Se propuso bancarizar al país a través del crédito usurario al consumo. Resultó un sistema de tiendas de raya con cartera vencida al alza. Se pretendió que, creando muchos bancos pequeños, se estimularía la competencia para bajar márgenes. Eso no ocurrió. Se generaron instituciones vulnerables sin base de depósitos que se encaminó a fondearse en el mercado. Cuando éste se cierra, las sofoles, sofomes, las instituciones de nicho, que concentran riesgo excesivamente tienen problemas.

2. Afortunadamente los grandes bancos están bien capitalizados y sólidos. Están a salvo con sus volúmenes de crédito mediocre, pero están actuando en forma procíclica, acentuando la baja de la actividad económica al endurecer las condiciones de crédito. Por ello el gobierno reimpulsa la banca de desarrollo.

3. Hay que repensar algunos elementos del modelo. Mediante políticas generales, las autoridades deben propiciar que la banca actúe en forma contracíclica, limitar el crédito al consumo, ampliarlo a la industria, agricultura y desarrollo regional; las tasas de interés no se fijan por decreto, pero deben guardar una relación razonable respecto del costo de captación; los grandes bancos deben cotizar obligatoriamente en la Bolsa Mexicana de Valores para mejorar su supervisión y debe inducirse un proceso de mexicanización gradual. Limitar la concesión de instituciones patito.

En suma, para Suárez Dávila, los bancos deben ser una palanca del desarrollo nacional. Retomar el concepto de que un banco debe cumplir un servicio de interés nacional. Cada institución debe definir ante sus consejos y las autoridades cómo están contribuyendo de manera explícita a los fines del desarrollo de México y su recuperación económica. Esa es la responsabilidad que implica manejar el ahorro de los mexicanos.

El 23 de enero el titular de la Secretaría de Hacienda y Crédito Público, Agustín Carstens, declara, ante la actual crisis financiera mundial, que el gobierno mexicano ha dispuesto una inyección de $400 \mathrm{mil}$ millones de pesos para el rescate de la economía mexicana: 200 mil millones contemplados en los dos planes anticrisis que ha puesto en marcha la administración calderonista -monto que equivale, a decir del funcionario, a $1.8 \%$ del Producto Interno Bruto-, y 200 mil más por concepto de fondos y garantías otorgados mediante la banca de desarrollo, impulso que, según 
Carstens, «hace 20 años no hubiera sido posible, y eso simplemente resalta las fortalezas que ha venido adquiriendo el país en estos años».

La presente coyuntura no sólo ha puesto en evidencia, a escala mundial, la voracidad y el carácter destructivo del modelo económico dominante, sino también las deficiencias de las autoridades mexicanas -formadas en la ortodoxia neoliberal- para manejar el rumbo financiero del país: cuando la crisis actual comenzaba a manifestarse en todo el orbe y a dar los primero signos de crudeza, el gobierno federal no pudo o no quiso articular un discurso de mínima congruencia con la situación, y demostró carecer de un mínimo de realismo para aceptar, primero, la existencia de la crisis, y para dimensionar, después, su tamaño y gravedad. A golpes de realidad, y ante el reconocimiento global de la necesidad de la intervención del sector público para corregir los efectos de los descalabros financieros, la administración federal se vio obligada a abandonar la inacción que la había caracterizado, pero lo hizo de manera por demás tardía y errática: con la puesta en marcha de medidas que resultan insuficientes, pues no alcanzan a corregir los efectos de otras que son ofensivas para la economía de millones de mexicanos -como es el alza de las gasolinas y el diesel-, con el mantenimiento de inercias, características de los gobiernos recientes, orientadas al salvamento de las grandes empresas y los capitales financieros, no al auxilio de los ciudadanos comunes.

Al día de hoy, en los paquetes de acciones anunciados por el gobierno no se prevén medidas para rescatar al campo, rubro por demás importante en un escenario de crisis alimentaria; tampoco se ven intenciones de avanzar hacia una política fiscal que dote de recursos al Estado sin ensañarse con los pequeños contribuyentes; no se vislumbra voluntad de los actores políticos y económicos para concluir la injusta política de contención salarial, ni se ha avanzado en el restablecimiento de los aparatos de bienestar y en el resarcimiento de las conquistas laborales que se han perdido en las últimas décadas.

En suma, no se ha hecho nada para corregir de fondo los problemas que han hecho vulnerable a México ante coyunturas mundiales como la actual, y que pasan por la aplicación de un modelo que ha derivado en un incremento de la pobreza, la marginación, la desigualdad; que ha potenciado la dependencia de la economía mexicana en relación con la estadounidense, y cuyos supuestos beneficios hoy quedan en entredicho, como lo demuestra el saldo negativo de la balanza comercial registrado durante 2008, que, de acuerdo con datos del Instituto Nacional de Estadística y Geografía (INEGI), asciende a 16838 millones de dólares, monto superior en más de $50 \%$ en relación con el obtenido en 2007 y que representa el peor déficit desde 1995. 
Ante estas consideraciones, es claro que los planes de rescate anunciados por el gobierno mexicano no serán de ayuda para el común de la población en tanto no se reoriente el rumbo de la economía nacional. ${ }^{29}$

Para Rogelio Ramírez de la $\bigcirc,{ }^{30}$ la estrategia mexicana de crecer si Estados Unidos crece y si no lo hace esperar a que lo haga, no tiene viabilidad hoy. En primer lugar porque la válvula de la emigración de medio millón de trabajadores por año no va a existir. En segundo lugar, porque México ya vive una crisis social por el alto desempleo y las carteras de crédito vencidas de la clase media, en combinación con un problema de falta de seguridad y violencia creciente. Los bancos mexicanos enfrentan un problema de carteras vencidas en tarjetas de crédito. Oficialmente esta cartera es de $9.9 \%$ del portafolio, pero el presidente de Banamex indicó que la cifra correcta para los seis bancos más grandes del país es de $16.2 \%$. También crece la cartera hipotecaria vencida y el gobierno se apresta a utilizar un crédito externo por $2 \mathrm{mil}$ 800 millones de dólares para el apoyo a la vivienda, como parte de los $90 \mathrm{mil}$ millones de pesos del fondo creado para el apoyo a las empresas mexicanas. Además, las pensiones van a resentir una fuerte pérdida de riqueza y por tanto mayor desconfianza y menor consumo. El potencial de protestas sociales contra la privatización de las pensiones va a crecer.

Para Ramírez de la $\bigcirc$ el gobierno de Felipe Calderón no tiene más opción que reconocer el problema actual con toda su crudeza. De inmediato tendría que suspender todas aquellas políticas que eran posiblemente consistentes con el modelo económico anterior y con la globalización, pero que no habían tenido resultados positivos para México, o bien los habían tenido negativos. Tendría que analizar si para las nuevas condiciones globales estas políticas tienen sentido y cómo hacer para que tengan efectos positivos en el empleo y la producción. Si no, debe cambiarlas; lo peor que podría hacer sería mantener políticas que perjudican el empleo o el producto sólo porque están basadas en la ideología. Por ejemplo, ha sido un gran error que en medio de esta crisis el gobierno haya reducido los aranceles de los productos que aún gozaban de protección, cuando esto no era una prioridad inmediata y cuando posiblemente dañe a muchas industrias que no pueden competir por otros costos altos, especialmente en servicios y energéticos.

Según él, no habrá suficientes recursos para dar apoyo financiero a todas las empresas que lo requieran. Tampoco hay para apoyar a las familias de clase media con sus deudas en tarjetas de crédito o a los

\footnotetext{
${ }^{29}$ La Jornada, 24 de enero, 2009.

30 Rogelio Ramírez de la O. «ìHacia un cambio de modelo económico en México?» Le Monde Diplomatic, México, número 5, enero, 2009.
} 
desempleados. Aunque existen programas sociales para las familias más pobres y es probable que continúen, la clase media quedará desprotegida. El deterioro en la calidad de vida será enorme, especialmente en las grandes ciudades que ya están agobiadas por problemas del crimen y la inseguridad, urbanismo, atropello de derechos ciudadanos y burocratismo en los servicios que prestan las autoridades. Los recursos que tiene el gobierno son finitos y se pueden agotar haciendo apenas los primeros rescates de empresas y manteniendo los programas sociales que ya están vigentes. Al mismo tiempo, es claro que cada sector va a sufrir pérdidas, el gobierno federal y los estatales, las empresas y los ahorradores. Lo urgente es que su proyecto procure distribuir las cargas entre sectores de manera equitativa, con la prioridad de mantener la actividad económica y el empleo.

Parte esencial de la redistribución de cargas requiere que el gobierno reduzca el gasto dispendioso y de poco impacto, que hoy puede llegar a 10\% de su presupuesto bruto de $300 \mathrm{mil}$ millones de dólares, y lo reasigne a proyectos verdaderamente urgentes. De manera simultánea tiene que plantear un programa de endeudamiento con el propósito de mantener proyectos prioritarios de gasto y preferiblemente de inversión pública.

Sin embargo, para el autor referido, nada de lo anterior se vislumbra como realista si antes el gobierno no sacude su ideología, dogmas y prejuicios. Y al hacerlo, también debe renovar su gabinete y sus equipos de trabajo, porque el equipo que aún cree en el paradigma viejo no podrá adaptarse a los cambios que deben tener sus estrategias y programas. Esto es lo deseable, pero podría ser imposible, si se toma en cuenta que Felipe Calderón apenas acaba de pronunciar un discurso en la reunión de Mercosur haciendo llamados al libre comercio en el mismo estilo de los discursos de los líderes políticos en la era de la globalización.

\section{LOS IMPACTOS SOBRE LA EMIGRACIÓN A ESTADOS UNIDOS}

Desde mediados de los años noventa del siglo anterior, proporcional a la profundidad de la crisis económica nacional, la emigración a los Estados Unidos ha crecido de forma constante hasta el 2006. Es así como se observa que en cada año del sexenio de Vicente Fox, unos 575 mil mexicanos migraron a Estados Unidos -más de 3 millones en total-, provocando el despoblamiento de centenas de comunidades en por lo menos 600 municipios del país, los cuales registran tasas negativas de crecimiento demográfico. Asimismo, en ese periodo aumentó el número de mujeres que abandonaron el país para buscar el sueño americano, colocándose en $45 \%$ del total. ${ }^{31}$

${ }^{31}$ La Jornada, 4 de marzo 2008. 
La tendencia creciente de la emigración a Estados Unidos se mantiene durante el primer año de gobierno de Felipe Calderón, ya que según datos del Consejo Nacional de Población, ${ }^{32}$ en 2007, 679611 mexicanos se establecieron en Estados Unidos.

Estas tendencias son ratificadas por el Banco Mundial ${ }^{33}$ que informa que en los últimos años han salido del país 644 mil 361mexicanos en promedio cada año. Así, México ha expulsado al $10 \%$ de su población.

El crecimiento constante de la emigración mexicana a los Estados Unidos en los últimos años se explica en gran medida por la buena situación económica de aquel país. Durante años, el flujo migratorio crecía cuando la salud de la economía norteamericana era robusta; disminuía ligeramente durante las recesiones; fluía hacia el norte durante la primavera, cuando hay empleos disponibles en el sector agrícola y en el de la construcción, y se dirigía al sur durante las fiestas decembrinas.

Sin embargo la tendencia reciente parece comenzar a cambiar, como lo indican los informes de la Patrulla Fronteriza estadounidense sobre cantidad de detenciones de indocumentados en la frontera norte y sus cercanías en los últimos tres años. En 2006 la cifra cayó 8\% a cerca de un millón. El 2007 descendió una quinta parte. El primer semestre del 2008 mostró un descenso de $17 \%$ en comparación con el periodo similar del año anterior. En resumen (y debido al inexacto registro de las detenciones fronterizas) el flujo migratorio es hoy alrededor de la mitad del torrente presenciado en 2000 , cuando se efectuaron un millón 640 mil detenciones. Estas cifras olvidan a quienes cruzan de manera satisfactoria y recuentan a los que fueron detenidos varias veces, pero muestra una clara tendencia. La misma que muestran las remesas. El Banco de México reporta que, después de años de crecimiento formidable están descendiendo los envíos de los migrantes en Estados Unidos. El año pasado, ese flujo de efectivo fue de 24 mil millones de dólares, superior al turismo. Pero, durante el primer trimestre de este año la cifra anual descendió $2.9 \%$, según un nuevo informe de Goldman Sachs.

Es probable que la evaluación de los flujos fronterizos después de 2001 exagerara el índice de crecimiento real. Pero aun así, es evidente que los migrantes en realidad están enviando menos dinero a casa. Una encuesta realizada en Estados Unidos publicada por el Banco Interamericano de Desarrollo en abril, confirmó que pocos hacen remesas con regularidad; en 2006 tres cuartas partes de los migrantes hacían envíos, este año sólo la mitad. No sólo es el caso de México; Brasil, segundo destino de remesas en la región, sufrió una disminución de $4 \%$ el año pasado a 7.1 mil millones de dólares.

\footnotetext{
32 Reforma, 24 de septiembre 2008.

${ }_{33}$ Milenio, 24 de enero 2008.
} 
Es probable que dos factores tan desagradables uno como el otro, expliquen el doble descenso en flujos de personas y dinero: la hostilidad hacia los migrantes, sobre todo indocumentados y la profundización de la crisis económica en Estados Unidos. El impacto del primer factor es evidente: leyes estatales que consideran ilegal emplear a migrantes sin documentos, redadas cada vez más agresivas a empresas que los contratan, y mejor tecnología para compartir información conducente a su arresto. La enorme inversión en defensas fronterizas es el ejemplo más visible. El Departamento de Seguridad presupuesta 12 mil millones de dólares para el siguiente año fiscal con el objeto de proteger la frontera contra personas en busca de trabajo (y los míticos terroristas que se encaminan a su objetivo) presupuesto que permite adquirir enormes cantidades de equipo, nueva tecnología y aumentar la construcción del muro fronterizo. Sin embargo, hostilidad y muros importarían menos si el panorama económico permaneciera sólido. Pero, la economía estadounidense parece deprimida, incluso si sortea una recesión. En mayo el desempleo se elevó a 5.5\%. La depresión en el sector inmobiliario y de la construcción -donde laboran muchos inmigrantes, sobre todo los recién llegados- ha sido en especial dolorosa. En junio, el Centro Hispanico Pew publicó un informe con cifras de desempleo de $7.5 \%$ entre inmigrantes, que se elevaban a $8.4 \%$ entre mexicanos y 9.3\% entre los que llegaron al país después de 2000. El año pasado, más de 220 mil migrantes perdieron sus puestos de trabajo en la construcción. Y los que tienen empleo ganan menos: los empleados latinos de la construcción experimentaron pérdidas salariales en 2007. ${ }^{34}$

Ante el impacto de la crisis financiera en Estados Unidos sobre el mercado laboral, en particular de la construcción, que durante todo el 2008 ha llevado al desempleo a más de 200 mil trabajadores, en México a partir del mes de octubre surge una creciente preocupación sobre el eventual retorno masivo de migrantes mexicanos desempleados de aquel país. Es así como se manifiestan diferentes posiciones sobre el eventual retorno masivo, la capacidad de la economía mexicana para absorberlos productivamente y la voluntad política de los gobernantes para diseñar planes integrales de reinserción a tales retornados.

Respecto al posible retorno masivo de migrantes, con frecuencia se manejan en la prensa mexicana cifras que no tienen ningún respaldo técnico para sustentarlas. Ejemplo de ellas son las declaraciones de la Asociación Mundial de Mexicanos en el Exterior ${ }^{35}$ a finales del mes de octubre, cuando manifiesta su presidente Carlos Villanueva, que en los próximos tres meses hasta un millón y medio de migrantes regresarán de Estados Unidos a

${ }^{34}$ Economist Intelligense Unit, La Jornada, 8 de julio 2008.

${ }^{35}$ El Universal, 31 de octubre 2008. 
México ante la crisis financiera en ese país del norte, «que de agosto a la fecha dejó sin empleo a casi un millón de mexicanos legales o

indocumentados». Villanueva expresa que la crisis ha generado un éxodo que pondrá a prueba al gobierno de Felipe Calderón para ofrecerles trabajo, educación y servicios, pues en su mayoría llegarán a comunidades en regiones pobres del país. La situación de la comunidad mexicana es dramática «cada quien vive su propio infierno, el sueño americano dejó de serlo». Calcula que 150 mil personas ya regresaron a sus lugares de origen en México según la información de sus 20 oficinas regionales en Estados Unidos.

Frente a la visión anterior, Renato Rosaldo, antropólogo de la Universidad de Stanford, sostiene que por el momento México no enfrentará una oleada de migrantes legales e ilegales que regresen a sus lugares de origen a causa de la crisis económica que padece Estados Unidos, debido a que al tratarse de una recesión mundial esa nación les ofrece mayores certezas de desarrollo que las que tendrán en su país. Considera infundada la existencia de un "pánico hispánico» en México por el probable regreso masivo de migrantes.

«Si la recesión es mundial no creo que regrese tanta gente, por que saben que no hay empleos ni aquí ni allá, por lo que buscarán la forma de sobrevivir en Estados Unidos. Buscar empleo es fundamental para los migrantes y es uno de los motivos por los que salieron de sus países de origen». Lo que si se puede predecir es que los que regresen en mayor número serán jóvenes solteros, porque, a diferencia de los que ya cuentan con familia, no están tan arraigados en Estados Unidos, no tienen redes sociales y carecen de hijos, añade Rosaldo. ${ }^{36}$

De los pocos trabajos con rigor académico sobre el posible regreso masivo de migrantes mexicanos al país, en la tercera semana de diciembre el Colegio de la Frontera Norte hace un aporte relevante con el trabajo «La crisis financiera en Estados Unidos y su impacto en la migración mexicana», ${ }^{37}$ el cual parte de señalar que la economía estadounidense comenzó a dar signos de desaceleración desde el 2006, especialmente en el sector de la construcción, y que la población latina e inmigrante ha sido afectada seriamente por esta transformación.

La crisis financiera estadounidense ha repercutido en una disminución del empleo en sectores claves para los inmigrantes mexicanos (construcción y manufactura de alimentos), un incremento del desempleo abierto de la población latina, una caída de los ingresos de los hogares de inmigrantes, y un creciente porcentaje de latinos que perciben un deterioro en sus condiciones de vida. Consecuentemente, la migración mexicana indocumentada empezó a mostrar síntomas de desaceleración desde el 2006 y, más recientemente, el

${ }^{36}$ La Jornada, 8 de diciembre 2008.

${ }^{37}$ La crisis financiera en Estados Unidos y su impacto en la migración mexicana, Colef, http://www. colef.mx/coyuntura/2.asp 17/12/2008

156 PRIMER SEMESTRE 2009 MIGRACIÓN Y DESARROLLO 
volumen de las remesas también inició una tendencia descendente que parecería revertirse debido a la depreciación del peso mexicano.

Dicho estudio destaca cómo la vulnerabilidad de los inmigrantes indocumentados no es resultado sólo de factores económicos, sino también de la ampliación de los operativos del gobierno de Estados Unidos que han conducido a la criminalización y deportación de miles de mexicanos. Es indudable que el ambiente económico, social y judicial es en estos momentos desfavorable y hostil hacia los migrantes mexicanos indocumentados en Estados Unidos.

Por otra parte, en ese estudio se señala cómo a pesar de que las condiciones de vida de los latinos y los inmigrantes se han deteriorado durante los últimos dos años, los datos de la Encuesta sobre Migración en la Frontera Norte de México (EmifNorte) no muestran ninguna evidencia de un retorno masivo de migrantes o de un patrón migratorio diferente a lo observado en años anteriores. Esto no refuta el hecho de que los migrantes que estén regresando a México para celebrar las fiestas decembrinas, como lo hacen tradicionalmente cada año, estén expresando su preocupación por el clima desfavorable al otro lado de la frontera. Aun más, es muy probable que muchos de los que no tengan arraigo familiar, empleo seguro y propiedad de una casa en Estados Unidos decidan quedarse en México en el corto plazo.

Los investigadores del Colef consideran que no se está dando aún un regreso masivo de migrantes mexicanos porque una parte de ellos están integrados plenamente a Estados Unidos, como lo demuestran los datos de encuestas nacionales en ese país. Un segundo factor se refiere al reforzamiento de la vigilancia fronteriza y el incremento de las redadas que elevan el costo de volver a México e intentar regresar a Estados Unidos como indocumentado. Un tercer factor es que estas mismas políticas, junto a cambios importantes en la demanda de trabajo inmigrante, han fracturado el patrón de migración circular que históricamente caracterizó al flujo México-Estados Unidos. Este patrón cambió principalmente desde 1986 por IRCA (Inmigration Reform and Control Act) al permitir el establecimiento de miles de familias en territorio estadounidense. Al verse forzados los migrantes indocumentados a permanecer más tiempo en Estados Unidos, los mexicanos han hecho migrar también a sus familias, lo que ha producido un mayor arraigo en dicho país. Un cuarto factor se refiere a la decisión de retornar a México que es muy compleja para millones de mexicanos indocumentados que cuentan con hijos que son ciudadanos estadounidenses o que asisten a la escuela en ese país. Un quinto factor trata la situación económica en México, que no es precisamente mejor que la de su socio comercial, por lo que el regreso no es un incentivo. Finalmente, existe una fuerte expectativa de reforma migratoria ante el triunfo electoral de Barack Obama. 
El planteamiento anterior es corroborado por el Instituto de Políticas Migratorias de Washington (MPI), ${ }^{38}$ que en la segunda semana de enero sostiene que no hay pruebas de una nueva tendencia de retorno a casa de mexicanos y latinoamericanos como resultado de la crisis aquí. Demetrios Papademetriou, responsable de la investigación sobre ese tema y director del Instituto, declara que «una migración sustancial de retorno de inmigrantes no autorizados es poco probable, a menos de que empeore severamente y por tiempo prolongado la economía estadounidense».

La investigación del MPI indica que más bien la migración de retorno parece tener una «mayor correlación con los acontecimientos económicos, políticos y sociales en los países de origen, que con las condiciones económicas en Estados Unidos».

Papademetriou considera que

mientras la actual crisis económica podría no percibirse como el momento más oportuno para corregir el desconecte crónico entre el mercado laboral estadounidense y el sistema de migración, los formuladores de políticas más visionarios reconocerán que un sistema más ágil y pensado servirá mucho mejor a los intereses económicos de Estados Unidos en un mercado global cada vez más competitivo.

Contrastando con el epígrafe anterior sobre el posible retorno de los migrantes mexicanos al país, el New York Times, ${ }^{39}$ informa de un nuevo flujo migratorio forzado del Centrooccidente de México a los Estados Unidos de los familiares de los migrantes a causa de la cantidad creciente de secuestros y extorsiones de que son objeto por parte de diferentes bandas delictivas como los «Zs», que han encontrado en esa región a la familia transnacional como un nuevo campo lucrativo para ejercer su actividad delictiva.

El mecanismo es secuestrar al familiar en las comunidades locales y exigirles a los migrantes en Estados Unidos el rescate vía electrónica. La extorsión constante en dinero y especie se ha incrementado sobre la familia migrante aprovechando la existencia de activos y remesas en esos hogares. En el artículo referido se dan testimonios de las comunidades de Felipe Ángeles y Los Haro en Zacatecas. Pero, existen múltiples evidencias de que lo mismo está sucediendo de forma creciente en Jalisco, Guanajuato y Michoacán, entre otras entidades del país.

Con este nuevo tipo de migración forzada por la inseguridad del país, entre otros efectos, puede crecer el despoblamiento de muchas de las comunidades y caer el interés de promover pequeños proyectos sociales y productivos como lo venían haciendo las organizaciones de migrantes en años anteriores.

Respecto a las propuestas de reinserción de los migrantes de 
retorno, es la gobernadora de Zacatecas, Amalia García, quien el 27 de octubre da a conocer ante el Conago (Consejo Nacional de Gobernadores) su propuesta y plantea que en el presupuesto federal del 2009 se considere una partida de 7,000 millones de pesos para realizar un plan nacional de reinserción de migrantes. ${ }^{40}$ Su propuesta contempla los siguientes aspectos:
a) Certificación de habilidades profesionales.
b) Fomento al autoempleo.
c) Programa $1 \times 1$ con migrantes retornados.
d) Capacitación laboral.
e) Alimentación básica.
f) Atención a salud.
g) Apoyo para compra de vivienda.

Por su parte, el 20 de octubre en el evento de la Secretaría de Relaciones Exteriores sobre la crisis financiera de Estados Unidos y su impacto sobre el futuro de la migración de México, ${ }^{41}$ los investigadores de la Universidad Autónoma de Zacatecas dieron a conocer su propia propuesta de reinserción integral de los migrantes de retorno, que contempla los aspectos siguientes:

a) Programa de microproyectos productivos familiares e individuales con apoyo integral.

b) Fomento de incubadoras transnacionales con la participación de las organizaciones de migrantes mexicanos en Estados Unidos.

c) Programa inmediato de reinserción educativa.

d) Selección y apoyo a ciudades medias con mayor capacidad de generar empleo.

e) Programa de rehabilitación de la infraestructura agropecuaria.

f) Programa de desarrollo local sustentable (tratamiento de basuras y aguas residuales y saneamiento ambiental).

g) Programa nacional de construcción de vivienda popular.

Finalmente, planteamos que lo esencial no es hacer un plan anticrisis sino un proyecto integral de transformación de la economía mexicana fuera de los cauces neoliberales que durante 26 años la han mantenido en un proceso de creciente estancamiento económico, extranjerización y empobrecimiento de la población nacional; en esta parte final lo relevante no radica en hacer un buen plan para la reinserción de los miles de migrantes que eventualmente puedan regresar a México a causa de la recesión económica en ese país, sino diseñar y aplicar una política de Estado sobre desarrollo y migración que permita colocar en el centro de todas las políticas públicas de México el desarrollo económico y social, el desarrollo de todas las regiones, la generación de empleo, el bienestar, la independencia, la democracia y la construcción de nuevas condiciones

${ }^{40}$ La Jornada, Zacatecas, 28 de octubre 2008.

${ }^{41}$ La crisis financiera en Estados Unidos y su impacto sobre el futuro de la emigración mexicana. Secretaría de Relaciones Exteriores. 
estructurales en la economía del país para que a mediano plazo los mexicanos puedan ejercer su derecho a no emigrar.

Una política de Estado sobre Desarrollo y Migración, entre otros elementos, debe contemplar los siguientes puntos: ${ }^{42}$

I. Que el Estado mexicano recupere su función como principal responsable y promotor del desarrollo económico nacional.

II. Que recupere su capacidad de planear el desarrollo económico y social de todas las regiones y sectores del país.

III. Que supere el falso paradigma del desarrollo con base en la mano invisible del mercado, aplicando políticas públicas que regulen y apoyen eficientemente el funcionamiento de todos los sectores económicos del país.

IV. Que se dé una coherencia entre las diferentes dependencias del gobierno federal como la Secretaría de Hacienda, el Banco de México, la Secretaría de Economía, la Secretaría de Desarrollo Social, la Secretaría de Agricultura, la Secretaría de Educación Pública y el Conacyt, para lograr el trabajo coordinado de fomento y respaldo al desarrollo económico integral con base al mercado interno, que sin descuidar la articulación con el mercado exterior, posibilite una estrategia de desarrollo de largo plazo con base en los recursos internos del país.
V. Que se reintegre la prioridad del desarrollo regional del país como una prioridad del desarrollo nacional. El auténtico desarrollo nacional se tiene que manifestar local y regionalmente en más y mejores empleos, bienestar y democracia para todos o no es realmente desarrollo económico nacional.

VI. El fracaso durante 25 años del mercado como promotor del desarrollo regional de México obliga a que la Secretaría de Economía reintegre el desarrollo regional como una prioridad hacia las PYMES de todo el país y que lo propio haga la Secretaría de Desarrollo Social y la Secretaría de Agricultura hacia las comunidades rurales, organizaciones sociales urbanas, los municipios y organizaciones no gubernamentales. Los graves problemas sociales del país muestran que la Secretaría de Desarrollo Social no puede seguir siendo el ministerio de la administración de la pobreza, como hasta ahora.

VII. En la Secretaría de Desarrollo Social se debe establecer la Subsecretaría de Desarrollo Regional que articule todas las propuestas de los estados y municipios sobre desarrollo regional. Para ello, debe aprovechar los diagnósticos y propuestas elaboradas por los fideicomisos regionales en el sexenio anterior como el Fideicomiso para el Centro

\footnotetext{
42 "Migración internacional y políticas públicas alternativas en México», en Cambiando perspectivas: de la gestión de flujos hacia la construcción de políticas de migración con enfoque de desarrollo. Sin Fronteras-Universidad Autónoma de Zacatecas-Incide Social-Miguel Ángel Porrúa, 2008.
} 
Occidente (Fiderco) que abordan los problemas regionales de forma integral, con la participación de varios estados, pero, que no llegaron a convertirse en política pública.

VIII. Si se busca realmente combatir a la pobreza y erradicar las causas estructurales de la emigración se requiere fortalecer la economía nacional, sus diferentes sectores y regiones, como se ha indicado antes. Pero, además, es necesario un cambio a fondo en la Secretaría de Desarrollo Social en términos de establecer un Sistema Nacional de Desarrollo Social, un Plan Nacional de Desarrollo Social, un Gabinete de Desarrollo Social y un Consejo Nacional de Evaluación independiente del Ejecutivo.

IX. Para que lo anterior se materialice en los hechos se requiere superar la paradoja planteada por Armando Bartra durante el salinato: «La política económica de Hacienda los empobrece y Sedesol mitiga su precariedad». Se necesita un cambio en la política económica actual que ha empobrecido a 50 millones de mexicanos, hacia una perspectiva neokeynesiana de crecimiento económico regional, crecimiento sostenido de empleos decorosos e impactos multiplicadores en la economía nacional con base a la inversión pública. Si la prioridad de la política social es la eliminación de la pobreza, la política económica debe ser coherente con tal prioridad, aplicando en forma conjunta estrategias de crecimiento económico regional y superación sostenible de la pobreza. Para ello el Banco de México, la Secretaría de Hacienda y la Banca de Desarrollo deben cambiar su forma de actuación en beneficio de unas cuantas grandes corporaciones nacionales y extranjeras, para recuperar las funciones para las cuales fueron creadas: fomento, apoyo, respaldo técnico y financiero para la mayoría de las empresas del país.

$\mathrm{X}$. Todo lo anterior supone un cambio radical en la clase política mexicana de poner en el centro el futuro económico, social y político del país. Las Cámaras de Diputados y Senadores no pueden seguir siendo testigos mudos $\mathrm{y}$ cómplices del empobrecimiento de la mayoría de la población, el enriquecimiento de unos pocos y la entrega de los activos nacionales a los extranjeros. Urge la desconstrucción de los partidos políticos actuales preocupados sólo por sus parcelas de poder, canonjías y mercado electoral, al margen de las necesidades, demandas y propuestas de la mayoría de los mexicanos. La discusión actual sobre la reforma del Estado es la oportunidad de que la clase política mexicana muestre si su compromiso es con las reformas del Estado hacia una etapa donde éste realmente funcione al servicio de las mayorías del país o si nuevamente es una simulación para seguir sirviendo a las oligarquías y a sus propios intereses. 